\title{
LAMPEDUSA TRA PRODUZIONE E RAPPRESENTAZIONE DEL CONFINE
}

\author{
Paolo Cuttitta ${ }^{7}$
}

Com'è avvenuta la trasformazione di Lampedusa in sinonimo della frontiera euro-africana? I confini sono fatti sociali, e trasformare un luogo in confine, o trasformare il ruolo simbolico e pratico di un confine, è sempre frutto di determinate azioni. Da questa prospettiva I'articolo analizza i principali atti che hanno accresciuto la "confinità" di Lampedusa, determinando la "frontierizzazione" dell'isola, tra gli anni Novanta e il 2011. L'analisi tocca alcune scelte del legislatore, l'evoluzione delle relazioni con i paesi nordafricani e certe pratiche di controllo e gestione delle frontiere: I'apertura di un centro di detenzione, la scelta di dirottare quasi tutti i migranti a Lampedusa, concentrandovi così anche le attività di accoglienza, e la produzione di emergenze artificiali. ${ }^{2}$

Parole chiave: Lampedusa, Italia, confine, migrazioni, controllo.

\section{Introduzione}

Ormai da anni, in Europa, Lampedusa è sinonimo di confine. Il suo nome evoca immagini di "sbarchi" e "tragedie del mare". Anche più di recente alcuni eventi eccezionali - la visita del papa, la strage del 3 ottobre 2013 - ne hanno confermato il ruolo (non solo simbolico) di frontiera per eccellenza.

Dal dicembre 2013, però, il centro di accoglienza dell'isola è rimasto chiuso per quasi un anno, in seguito alla rescissione del contratto tra il governo e I'ente gestore a causa del maltrattamento degli ospiti. Di conseguenza, proprio nel 2014 (quando gli arrivi sulle coste italiane quasi triplicano rispetto al precedente record del 2011), I'incidenza degli sbarchi a Lampedusa crolla bruscamente. Nemmeno la riapertura del centro ha ripristinato la situazione precedente: i

1 Ricercatore presso la Vrije Universiteit di Amsterdam, Faculty of Law, Amsterdam, Olanda.

2 Il corpo principale di questo articolo è la rielaborazione della prima parte del seguente volume: CUTTITTA, Paolo. Lo spettacolo del confine. Lampedusa tra produzione e messa in scena della frontiera. 
migranti intercettati nel Canale di Sicilia continuano infatti a essere condotti in altri porti. Lampedusa continua tuttavia a essere la più usata (e abusata) sineddoche non solo del confine ma del fenomeno migratorio in generale. Un convegno organizzato a novembre 2014 da un prestigioso istituto di ricerca, per esempio, recava il titolo The "Lampedusa dilemma": Global flows and closed borders What should Europe do? Esso affrontava il tema dell'immigrazione irregolare e dell'asilo in Europa in relazione a variabili quali gli sviluppi demografici, il mercato del lavoro e gli obblighi di protezione. Lampedusa - che in quello stesso mese di novembre vedeva transitare appena 86 persone, mentre oltre novemila ne giungevano in altre località come Porto Empedocle, Pozzallo e Augusta - era usata come sinonimo non solo della frontiera marittima italiana ma dei confini europei in generale.

II grado di "confinità"3 di un determinato luogo in un determinato contesto storico è sempre - almeno in certa misura - effetto della sua collocazione geografica. Pertanto non meraviglia il fatto che Lampedusa sia molto più "confine" di tanti altri luoghi di confine italiani ed europei, più distanti da paesi terzi e rotte migratorie. Il solo contesto geografico e storico, tuttavia, non basta a spiegare perché Lampedusa sia più "confine", per esempio, delle coste della Calabria orientale o della Sicilia occidentale, e perfino di Pantelleria, che pure si trova più vicina all'Africa.

Per rispondere a tale interrogativo bisogna ricordare le parole di un antesignano degli studi sui confini: il confine "non è un fatto spaziale con effetti sociologici, ma è un fatto sociologico che si forma spazialmente" ${ }^{4}$. Anche oggi i Border Studies si propongono di de-naturalizzare i confini, rivolgendo la propria attenzione ai relativi processi costitutivi ${ }^{5}$ e partendo dal presupposto che "tutti i confini sono un prodotto della storia e della società" ${ }^{.}$.

Insomma, trasformare un luogo in confine, o modificare il significato simbolico o il ruolo pratico-operativo di un dato luogo di confine, è sempre il risultato di determinati atti. Questo articolo illustra i principali tra gli atti che, dagli anni Novanta al 2011, hanno determinato la "frontierizzazione"7 di Lampedusa, creando la frontiera Lampedusa e innalzandone il tasso di "confinità"8.

3 Il termine "confinità" esprime l'insieme di quelle caratteristiche che fanno di un luogo un confine.

4 SIMMEL, Georg. Sociologia, p. 531.

5 VAN HOUTUM, Henk; VAN NATERS, Ton. Bordering, Ordering and Othering.

6 DELL'AGNESE, Elena. Geografia politica critica, p. 102.

7 Con il verbo "frontierizzare" e il suo derivato "frontierizzazione" si esprime l'azione di trasformare qualcosa in confine.

8 Analogamente si potrebbero analizzare altri luoghi simbolo del confine europeo come Ceuta, Melilla, Calais e I'Evros. 


\section{Alle origini della "confinità"}

Le radici dell'attuale "confinità" di Lampedusa sono da rinvenire, innanzitutto, nelle misure restrittive in materia di immigrazione adottate a partire dal 1990, quando I'Italia aderisce agli accordi di Schengen: è allora che viene varata la prima legge organica sull'immigrazione (la seconda sarà approvata subito dopo l'entrata in vigore degli accordi stessi) ${ }^{9}$. Mi riferisco in particolare ai due più classici strumenti di controllo dell'immigrazione. Il primo è l'imposizione dell'obbligo del visto ai cittadini della maggior parte dei paesi extra-UE. Il secondo è l'imposizione di sanzioni ai vettori (compagnie di trasporto aereo, terrestre o marittimo) che trasportino in un determinato paese passeggeri privi dei documenti richiesti per l'ingresso. Tali misure danno al fenomeno dell'immigrazione irregolare le sue attuali forme.

Infatti, se chi sceglie di imbarcarsi (irregolarmente) alla volta di Lampedusa non fosse sottoposto all'obbligo del visto, potrebbe viaggiare regolarmente con il solo passaporto. Chi volesse raggiungere l'Europa per chiedervi asilo, cercarvi lavoro o ricongiungervisi con i propri cari (o semplicemente per la curiosità di vedere com'è fatta!) potrebbe farlo con regolari mezzi di linea, e non sbarcherebbe a Lampedusa. L'ingresso irregolare, perciò, sarebbe un'opzione solo per chi non fosse in grado di ottenere un passaporto dalle autorità del proprio paese. Ma anche in presenza dell'obbligo del visto le compagnie di trasporto, se solo non temessero di incorrere in sanzioni, continuerebbero ad accogliere a bordo tutti gli acquirenti di un biglietto. Ormai, invece, i loro dipendenti agiscono quasi come guardie di frontiera private, con la differenza che, mentre le vere guardie di frontiera non possono (almeno teoricamente) impedire l'ingresso nel territorio a chi cerchi asilo, le compagnie di trasporto non sono tenute da alcuna legge ad accogliere a bordo rifugiati.

Senza l'adozione di tali misure, insomma, le migrazioni irregolari via mare sarebbero un fenomeno residuale se non inesistente, e Lampedusa non sarebbe il simbolo della frontiera euro-africana.

\section{Concentrare i migranti, concentrare il confine}

\section{I rapporti con Tunisia e Libia}

La quota dei migranti approdati a Lampedusa in rapporto all'intero territorio siciliano è relativamente bassa fino al 2001: il 18\% nel 1999, meno del 17\% nei due anni seguenti. D'improvviso, però, essa supera il 53\% nel 2002. La crescita è di oltre 36 punti percentuali in un solo anno. Dal 2003 al 2008

${ }_{9}$ Legge 39 del 28 febbraio 1990 (legge Martelli); legge 40 del 6 marzo 1998 (legge TurcoNapolitano). 
(quando sfiora I'89\%) la quota degli sbarchi a Lampedusa sul totale degli arrivi in Sicilia rimane sempre sopra il 60\% (nel 2009 e nel 2010, invece, si nota un brusco calo, dovuto ai respingimenti effettuati dal Canale di Sicilia verso la Libia, cui si fa cenno più avanti).

\begin{tabular}{|c|c|c|c|c|c|}
\hline \multicolumn{5}{|c|}{ Migranti giunti irregolarmente alle frontiere marittime italiane } \\
\hline Anno Elaborazione dell'autore di dati del Ministero dell'Interno e, per il 2014, della Fondazione Migrantes)
\end{tabular}

Tale dato - coincidente peraltro con un aumento dei valori assoluti degli sbarchi in Sicilia - non è l'effetto di un aumento delle partenze dalle coste libiche (dalle quali è più facile raggiungere Lampedusa che altre coste siciliane), a sua volta dovuto a un incremento delle migrazioni subsahariane. Nel 2002, infatti, almeno parte dei subsahariani continua a partire dalla Tunisia e arriva sulle coste siciliane. Del resto, anche se i 5.385 subsahariani arrivati nel 2002 fossero partiti tutti dalla Libia giungendo tutti a Lampedusa, il loro numero, da solo, sarebbe ben lungi dallo spiegare l'incremento degli sbarchi a Lampedusa sul totale degli sbarchi in Sicilia.

Già dagli anni Novanta i subsahariani partono, proprio come i ben più numerosi maghrebini, dalle coste tunisine. Queste, infatti, sono più vicine di quelle libiche a Lampedusa, a Pantelleria e alla Sicilia. Inoltre, in Tunisia esistono già organizzazioni consolidate che gestiscono le traversate. Le partenze dalla Libia (e gli arrivi di subsahariani) aumentano, sì, nel corso degli anni Zero, ma in primo luogo ciò non avviene in tempi e con modalità tali da spiegare il "grande balzo in avanti" del 2002, e in secondo luogo questo loro incremento non è una fatalità: le cause che lo determinano sono riconducibili più o meno direttamente all'azione del governo italiano.

Una prima causa può essere la conclusione, nell'estate del 1998, del primo accordo di riammissione con la Tunisia ${ }^{10}$ : il regime di Ben Alì s'impegna a

${ }^{10}$ CUTTITTA, Paolo. I confini d'Europa a Sud del Mediterraneo. Strumenti e incentivi per l'esternalizzazione dei controlli. 
riaccogliere nel proprio territorio non solo i propri cittadini giunti o soggiornanti irregolarmente in territorio italiano, ma anche i cittadini di altri paesi (esclusi i maghrebini) giunti irregolarmente in Italia passando dalla Tunisia. S'intensifica così la persecuzione dei subsahariani. Già in precedenza i migranti "africani" (così gli arabi nordafricani usano definire i neri) erano vittime, nel paese di Ben Alì, di vessazioni e di deportazioni sommarie e indiscriminate. Ma la formalizzazione dell'obbligo a riammettere queste persone, e quindi a doversene fare carico, qualora esse giungano irregolarmente in Italia dalla Tunisia, convince evidentemente le autorità tunisine a inasprire l'azione repressiva nei loro confronti ${ }^{11}$. La Libia diventa allora un luogo d'imbarco alternativo per molti.

Una seconda causa è certamente la contestuale svolta impressa dal governo italiano alle relazioni con la Libia. Quando il Ministro degli Esteri italiano Dini incontra, nel 1998, I'omologo libico El-Muntasser, ed esprime a nome dell'Italia il "rammarico per le sofferenze arrecate al popolo libico a seguito della colonizzazione"12, tra i propositi vi è anche quello di avviare una cooperazione nel settore "della prevenzione e lotta all'immigrazione clandestina"13. Tale collaborazione avrà inizio in seguito alla firma, nel 2000, del primo accordo di cooperazione di polizia. Gheddafi, così, si trova nelle mani un jolly che utilizzerà con sapienza, alzando il prezzo dell'amicizia con l'ex potenza coloniale e ottenendo benefici economici e politici dall'Italia e dall'Europa ${ }^{14}$. Il leader libico non esita infatti ad allentare o rafforzare i controlli, a favorire o a ostacolare le partenze dalle coste del proprio paese in funzione dei propri interessi. Nei primi anni del decennio, in particolare, Gheddafi preme sull'Italia affinché I'Unione europea cancelli l'embargo contro la Libia. L'obiettivo è raggiunto a fine settembre del 2004, e solo allora Gheddafi accetta di riammettere migliaia di migranti partiti dalla Libia: è l'inizio dei respingimenti del 2004-2006 ${ }^{15}$. Il ricatto del Colonnello continua, poi, anche negli anni seguenti. L'obiettivo è ottenere dall'Italia adeguate compensazioni per il periodo coloniale, e ciò avviene nel febbraio 2009 con la ratifica italiana del trattato di amicizia del $2008^{16}$. Nel 2011, infine, Gheddafi fa partire, per rappresaglia contro le bombe della Nato, decine di migliaia di "africani" verso Lampedusa ${ }^{17}$.

11 DEL GRANDE, Gabriele. Mamadou va a morire: La strage dei clandestini nel Mediterraneo, p. 116120.

12 AIRL.IT. Comunicato congiunto.

13 AIRL.IT. Processo verbale.

${ }^{14}$ CASSARINO, Jean-Pierre (a cura di). Unbalanced Reciprocities. Cooperation on Readmission in the Euro-Mediterranean Area; PAOLETTI, Emanuela. The Migration of Power and North-South Inequalities: The Case of Italy and Libya.

15 Tra ottobre 2004 e marzo 2006 oltre duemila migranti vengono respinti in Libia da Lampedusa, più di mille da altre località italiane.

${ }_{16}$ RONZITTI, Natalino. II trattato Italia-Libia di amicizia, partenariato e cooperazione.

17 FORTRESSEUROPE.BLOGSPOT.IT. Deportati in Italia. La mano del ra'is dietro gli sbarchi. 


\section{II "sistema" Lampedusa}

Queste considerazioni aiutano a comprendere come Lampedusa diventi il luogo di sbarco privilegiato nel decennio scorso ma non spiegano il "grande balzo in avanti" del 2002. La prima spiegazione di tale fenomeno è l'evoluzione del sistema di ricettività di Lampedusa. Nei primi anni Novanta, quando gli sbarchi sono ancora sporadici, non esiste ancora, sull'isola, una struttura di accoglienza. Il sistema dei pattugliamenti e dei controlli non si è ancora sviluppato, e spesso i rari migranti arrivano autonomamente sull'isola, senza essere visti, e cercano riparo in abitazioni abbandonate o incustodite. Altrimenti le autorità li ospitano in qualche rifugio improvvisato. Anche la fornitura di cibo e vestiti è lasciata al buon cuore degli abitanti e delle istituzioni civili e religiose locali. I tempi di permanenza sull'isola sono comunque minimi: per lo più i migranti prendono, dopo meno di ventiquattr'ore, il primo traghetto per la Sicilia. Loro non hanno motivo di restare, e nessuno ha interesse a che il loro soggiorno si prolunghi.

A metà degli anni Novanta gli arrivi s'intensificano: nel 1995 si contano 55 sbarchi per un totale di 1.227 persone, nel 199670 sbarchi e 2.778 persone. Viene allora aperto il primo centro di accoglienza, dotato di una capienza di 186 posti. I trasferimenti continuano a essere immediati. Quando però, nel 1998, la legge Turco-Napolitano introduce la detenzione amministrativa, il centro di Lampedusa, che formalmente resta un semplice centro di accoglienza, comincia occasionalmente a svolgere di fatto anche funzioni di centro di detenzione. II trattenimento prolungato nella struttura lampedusana diventa invece prassi abituale proprio dal 2002, quando la legge Bossi-Fini prolunga la durata massima del trattenimento nei Cpt da 30 a 60 giorni.

Di fatto, quindi, a partire dal 2002 il centro di Lampedusa non è più (solo) un centro di accoglienza ma anche (se non soprattutto) un centro di detenzione. Anche per questo la struttura è molto spesso sovraffollata. Benché i migranti, privati della possibilità di uscire, siano quasi invisibili, la loro presenza stabile e massiccia comincia a cambiare il volto dell'isola, dandole le sembianze della frontiera. Che il centro, a fronte di una capienza massima di 186 persone, ne ospiti solo qualche decina oppure cinquecento o addirittura settecento (come nell'agosto 2005) non è la stessa cosa. La prassi di usare il centro di accoglienza come centro di detenzione e di aumentare a dismisura sia il numero dei trattenuti, sia i tempi di trattenimento, si afferma a partire dal 2002 e si conferma fino al $2006^{18}$.

Ma nel 2002 cambia anche la gestione della struttura. Fino ad allora essa è affidata alla Croce rossa, che fruisce del lavoro gratuito di decine di volontari.

\footnotetext{
$\overline{18}$ Nel febbraio 2006 il governo chiarisce che la struttura è un centro di accoglienza. Da allora, per più di due anni, i tempi di trasferimento si riducono notevolmente.
} 
Nel 2002, invece, la gestione passa alla confraternita della Misericordia, e cambiano i termini dell'accordo. Nel centro adesso opera personale stipendiato, e il Ministero dell'Interno eroga all'ente gestore una determinata cifra per ogni giorno di permanenza di ogni ospite. Al volontariato dell'accoglienza si sostituisce così il professionismo della gestione delle frontiere. Ai soldi destinati direttamente alla gestione della struttura si aggiunge inoltre il giro d'affari legato alla presenza di militari e altri operatori. L'interesse economico del gestore a ospitare più migranti, e per periodi più lunghi, si sposa con l'interesse politico del governo, che può concentrare "in un luogo piccolo e distante l'emergenza immigrazione". A Lampedusa, quindi, sono portati anche migranti intercettati a cento miglia dall'isola e diretti da tutt'altra parte, per evitare "che sbarchino in Sicilia"19.

II "sistema" così costruito a Lampedusa è unico nel suo genere: su nessun'altra isola minore italiana esiste un centro stabilmente operativo e dotato di personale dipendente. Anche a Pantelleria, I'isola italiana più vicina all'Africa, si appronta, già negli anni Novanta, un centro di accoglienza informale, ma la struttura non acquisirà mai le caratteristiche della stabilità. Da quando si formalizza il centro di Lampedusa, quello di Pantelleria perde importanza fino, di fatto, a scomparire, per essere temporaneamente ripristinato solo in circostanze eccezionali.

In tale contesto s'inserisce, nello stesso 2002, I'annuncio del secondo governo Berlusconi di volere intensificare i controlli delle frontiere marittime con mezzi militari aerei e navali, estendendo le relative attività anche alle acque internazionali. In realtà le acque internazionali sono pattugliate già dal $1995^{20}$. Tuttavia l'intensificazione dei pattugliamenti potrebbe spiegare - seppur solo in parte - l'aumento generale del numero di persone fermate alle frontiere marittime italiane. È probabile, cioè, che dal 2002 si cominci a intercettare più al largo delle coste italiane anche una parte di coloro i quali, altrimenti, morirebbero lungo il viaggio o, approdando sulle coste siciliane, si dileguerebbero senza essere notati. Ma, soprattutto, aumentando gli intercettamenti in acque internazionali aumenta anche il potere di decidere dove fare sbarcare i migranti. E la stragrande maggioranza di quelli che vengono intercettati, dal 2002 in avanti, viene portata a Lampedusa, ad alimentare il funzionamento del "sistema" che si sta consolidando.

In definitiva, il centro di accoglienza/detenzione di Lampedusa ha un triplice ruolo nel processo di "frontierizzazione" dell'isola. In primo luogo esso

${ }^{19}$ Da un'intervista a Giusi Nicolini (poi eletta sindaco di Lampedusa e Linosa nel 2012) in LIBERTI, Stefano. A sud di Lampedusa. Cinque anni di viaggi sulle rotte dei migranti, p. 189-191.

${ }^{20}$ CAMERA.IT. Comitato parlamentare Schengen-Europol. Sopralluogo a Lampedusa (18-19 e 20 luglio 1997). 
consente il trattenimento prolungato di elevati numeri di migranti. In secondo luogo, le autorità italiane vi dirottano quasi tutti i migranti intercettati nel Canale di Sicilia, almeno una parte dei quali, altrimenti, approderebbe altrove. Infine, la gestione di una simile struttura e di un più elevato numero di sbarchi attrae sull'isola un gran numero di persone. L'identità di un luogo è data innanzitutto da chi ci vive, ci lavora o ci transita. Mentre le altre isole minori siciliane sono popolate solo da residenti stabili dediti al turismo e alla pesca e, su base stagionale, da turisti, Lampedusa è popolata in modo consistente (e più o meno stabilmente) anche da varie categorie di persone dedite ad attività legate al controllo e alla gestione della frontiera: carabinieri, finanzieri e guardie costiere; agenti di polizia italiani e stranieri; funzionari comunitari dell'agenzia Frontex; medici, mediatori culturali, operatori legali e umanitari di organizzazioni non governative nazionali e internazionali; politici italiani ed europei che vengono a ispezionare il centro di detenzione, a trattare con la popolazione o a sfruttare la visibilità dell'isola per fare campagna elettorale; operatori dei media arrivati per informare sul fenomeno del controllo dell'immigrazione; ricercatori e artisti venuti per studiarlo e interpretarlo; attivisti giunti per criticarlo; etc. Ciò comporta - insieme all'aumento del numero di migranti e dei tempi di loro permanenza - anche una radicale trasformazione della composizione della popolazione, del paesaggio umano dell'isola, e fa aumentare vertiginosamente insieme al fatturato dell'industria della frontiera ${ }^{21}$ - anche il grado di "confinità" di Lampedusa.

\section{Cambi di rotta: la svolta tunisina}

Un altro fattore determinante per la "frontierizzazione" di Lampedusa è la svolta collaborativa della Tunisia, collocabile nel 200422. Fino ad allora la Tunisia è ancora la principale base di partenza, e le rotte puntano anche verso la Sicilia e Pantelleria.

La svolta comincia nel dicembre del 2003, quando il regime di Ben Alì, in cambio di adeguate contropartite, firma il secondo accordo di cooperazione con I'Italia (quello del 1998 non aveva dato i frutti sperati in Italia). Conseguentemente la Tunisia adotta una serie di misure che colpiscono sia i propri cittadini, sia gli stranieri in transito verso l'Italia. La riforma del codice di commercio marittimo e del codice di navigazione marittima mira a scoraggiare l'uso di imbarcazioni locali per le traversate. La riforma della legge sui passaporti introduce pesanti pene per ogni attività legata alle migrazioni irregolari. Contemporaneamente s'intensificano

${ }^{21}$ FRIESE, Heidrun. Border Economies. Lampedusa and the Nascent Migration Industry; RODIER, Claire. Xénophobie business. A quoi servent les contrôles migratoires?

22 BOUBAKRI, Hassan. La Tunisia nel contesto euro-maghrebino. Controllo dei movimenti migratori e diritti dei migranti. 
notevolmente i controlli sulle coste. Se fino ad allora partire dai porti tunisini era relativamente semplice, dal 2004 le probabilità di essere intercettati già alla partenza o in acque territoriali tunisine aumentano enormemente.

Ciò spinge i migranti (tunisini e non) oltre il confine libico. Abbandonate quasi del tutto le rotte tunisine (riprese, poi, solo nel 2011, quando la rivoluzione fa saltare i controlli), si intensificano le partenze dalla Tripolitania, da dove Lampedusa è di gran lunga l'obiettivo più vicino. Ciò spiega il sensibile aumento degli arrivi sull'isola nel 2004: la percentuale degli sbarcati a Lampedusa sul totale degli arrivi in Sicilia cresce di quattordici punti percentuali rispetto all'anno precedente, e rimane fino al 2008 al di sopra dei valori del $2003^{23}$.

La svolta tunisina suggerisce, infine, un'ulteriore riflessione. L'adozione delle nuove leggi e il rafforzamento del sistema di sorveglianza delle frontiere marittime sono atti mal visti dalla popolazione tunisina, e lo stesso governo è riluttante a compierli. Le rimesse degli emigrati, infatti, costituiscono una delle principali risorse dell'economia del paese. Se il governo tunisino, quindi, decide di imporre questa svolta restrittiva, ciò si deve alle pressioni esercitate e alle contropartite politiche, tecniche ed economiche offerte dall'Italia ${ }^{24}$. II decremento degli arrivi dalle coste tunisine e il contestuale aumento degli arrivi dalle coste libiche (e quindi l'aumento degli sbarchi a Lampedusa) possono perciò esser fatti risalire, in ultima analisi, all'azione delle autorità italiane ${ }^{25}$.

\section{Acuire le crisi, aumentare la "confinità"}

Certi problemi - spiega Edelman ${ }^{26}$ - sono appositamente costruiti per giustificare l'adozione di determinate soluzioni e legittimare l'aumento di potere dei regimi. Le crisi inscenate a tal fine dagli stati, poi, si manifestano spesso "ai margini geografici del territorio nazionale: nelle isole, negli aeroporti, nei mari e nei centri di detenzione offshore, dove autorità e migranti si incrociano" 27 . Lampedusa ne è un esempio.

Se il carattere di "confinità" di un luogo non è solo legato al ruolo che quel luogo riveste nel campo del controllo e della gestione dell'immigrazione ma dipende anche dalla maggiore o minore problematicità con la quale il fenomeno migratorio vi si manifesta, allora acuire tale problematicità equivale a innalzare il tasso di "confinità" del luogo stesso. Se Lampedusa fosse sempre e solo un luogo

\footnotetext{
${ }^{23}$ Un altro fattore è peraltro da individuare in un fenomeno analogo, seppur meno rilevante quantitativamente: la collaborazione dell'Egitto nel controllo del Canale di Suez, avviata alla fine del 2003, che spinge diversi migranti orientali verso la rotta libico-lampedusana.

${ }^{24}$ CUTTITTA, op. cit.

${ }^{25}$ Anche la collaborazione dell'Egitto (v. nota 23) è ottenuta solo a prezzo di consistenti contropartite.

${ }^{26}$ EDELMAN, Murray. Constructing the Political Spectacle.

${ }^{27}$ MOUNTZ, Alison. Seeking Asylum. Human Smuggling and Bureaucracy at the Border, p. xvii.
} 
di transito ben organizzato ed efficiente (e i migranti accolti decorosamente e celermente trasferiti altrove) la "confinità" dell'isola sarebbe ridimensionata da una realtà locale ordinata e pacifica. L'isola, invece, vive diversi momenti di elevata criticità che la proiettano al centro dell'attenzione politico-mediatica, innalzandone vertiginosamente il tasso di "confinità". I due casi più emblematici si verificano nel 2009 e nel 2011.

Alla fine del 2008 il nuovo governo decide di rallentare il ritmo dei trasferimenti proprio nel momento in cui aumentano gli arrivi (soprattutto di tunisini). Il centro di accoglienza, quindi, si riempie all'inverosimile. La capienza ufficiale è di 381 persone (804 in situazioni di emergenza) ma gli ospiti sono 1.560, I'11 novembre, e 1.572, il 28 dicembre. II 30 dicembre il governo annuncia che - d'ora in avanti - chiunque arrivi sarà trattenuto sull'isola a tempo indeterminato fino al rimpatrio. Tra novembre 2008 e marzo 2009 centinaia di persone sono fermate più a lungo anche dei sessanta giorni previsti dalla legge per i centri di detenzione. In realtà la struttura è tornata a essere, dal 2006, un centro di accoglienza, ma a gennaio 2009 il governo ne dichiara la conversione in centro di detenzione, e annuncia la costruzione di un secondo centro. Ciò scatena la reazione della popolazione lampedusana, che il 23 gennaio organizza una manifestazione di protesta. Quello stesso giorno, molti dei 1.840 migranti detenuti nel centro vengono incredibilmente (ma non inspiegabilmente) lasciati liberi di uscire. I telegiornali mostrano le immagini della protesta dei lampedusani e quelle di centinaia di migranti che sciamano per Lampedusa. Le autorità rispondono inviando centinaia di poliziotti, carabinieri e guardie di finanza. A fine gennaio se ne contano quasi uno per ogni dieci abitanti: la militarizzazione dell'isola ne sugella la "frontierizzazione". L'avvio di una serie di respingimenti verso la Tunisia fa esplodere la protesta dei migranti detenuti nel centro, che il 18 febbraio è incendiato e semidistrutto.

La crisi del 2009 contribuisce a definire l'immigrazione via mare come problema di ordine pubblico e, quindi, a giustificare l'adozione di misure eccezionali, come l'allungamento dei tempi massimi di trattenimento nei centri di detenzione da due a sei mesi ${ }^{28}$ e l'avvio dei respingimenti collettivi verso la Libia. I pattugliamenti congiunti italo-libici, annunciati a marzo, cominciano il 6 maggio; da quel giorno oltre 1.400 persone, intercettate in acque internazionali, sono riportate in Libia illegittimamente ${ }^{29}$.

All'inizio del 2011, invece, la struttura è chiusa e vuota da mesi, perché i respingimenti verso la Libia e la collaborazione di Gheddafi nel fermare le partenze

\footnotetext{
${ }^{28}$ Il provvedimento è preso il 20 febbraio 2009.

29 Il 23 febbraio 2012 la Corte europea dei diritti dell'uomo condanna I'Italia nell'unico caso in cui un gruppo di migranti sia riuscito a presentare ricorso.
} 
hanno ridotto gli arrivi in acque italiane. Nel periodo 2009-2010, inoltre, i pochi migranti intercettati dalle autorità italiane in acque nazionali sono condotti in altri porti siciliani. Così Lampedusa può essere utilizzata mediaticamente anche quando è vuota: mostrare il centro chiuso e l'isola priva di migranti significa dimostrare che il governo ha azzerato gli sbarchi. Perciò si esita a riaprire la struttura anche davanti all'evidenza di arrivi sempre più numerosi, propiziati dalla Primavera araba e dalla caduta di Ben Alì il 14 gennaio. II mancato trasferimento dei migranti trasforma l'isola in un campo a cielo aperto. Il 12 febbraio sono circa 4.000 i migranti costretti a dormire per le strade. Solo allora si proclama lo stato di emergenza umanitaria, e si riapre il centro di Lampedusa. Cominciano (ma procedono lentamente) anche i trasferimenti. A fine marzo sono presenti 6.200 migranti: più che i residenti dell'isola. Alcuni sono stipati nel centro o in altre strutture improvvisate, ma tanti sono letteralmente senzatetto. La crisi, anche a causa della guerra in Libia, si protrae per tutta l'estate, finché un nuovo incendio appiccato dai migranti comporta la chiusura del centro di detenzione. Il governo dichiara Lampedusa "porto non sicuro", e il centro sarà riaperto solo nell'estate 2012 (ciò spiega la riduzione degli arrivi nel 2012).

Anche in questo caso la situazione di crisi provocata a Lampedusa contribuisce a rafforzare l'interpretazione emergenziale e sicuritaria del fenomeno migratorio, favorendo l'adozione di misure eccezionali: il 23 giugno 2011 il termine massimo di detenzione amministrativa è prolungato a diciotto mesi, mentre a fine settembre l'isola viene evacuata per mezzo di navi che, per i sette giorni durante i quali errano senza meta per il Mediterraneo, diventano il nuovo domicilio coatto di migliaia di persone, trasformandosi in centri di detenzione itineranti, al di fuori di ogni previsione giuridica ${ }^{30}$.

\section{Respingere i migranti, dilatare il confine}

Nel 1998 la legge Turco-Napolitano introduce una misura - il cosiddetto respingimento differito - che dilata operativamente la linea del confine, trasformando Lampedusa in una "zona di frontiera". Per capire cosa ciò significhi è necessario, innanzitutto, spiegare la differenza tra respingimento ed espulsione.

I cittadini stranieri che provano a entrare irregolarmente in territorio italiano sono soggetti a respingimento. Poiché viene loro negato il passaggio della linea di confine, si dice che essi sono respinti alla frontiera. Coloro i quali vengono individuati in posizione irregolare nel territorio ricevono, invece, un ordine di espulsione. All'ordine di espulsione sono collegati alcuni diritti, tra cui quello di presentare ricorso; con il ricorso viene sospesa l'esecutività

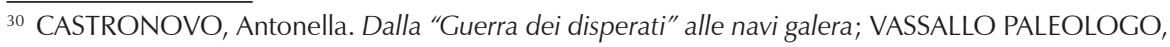
Fulvio. Diritti sotto sequestro, p. 191-196. 
dell'ordine di espulsione, e il destinatario può così trattenersi legittimamente in territorio italiano fino alla sentenza. Al contrario, il diritto di appellarsi contro un respingimento non comprende la sospensiva della misura; questa, pertanto, è comunque eseguita nelle more del giudizio (peraltro è rarissimo che un respinto abbia effettivamente possibilità di fare ricorso).

La particolarità della legge del 1998 consiste nel suo estendere I'applicabilità del respingimento - che normalmente si rivolge a chi sia trovato privo di documenti alla frontiera, cioè lungo la linea del confine - anche a chi sia intercettato subito dopo avere attraversato il confine. Poiché il termine "subito" non ha un significato preciso, le autorità di polizia hanno un ampio margine discrezionale in relazione alla distanza spazio-temporale rispetto al punto e al momento in cui il confine è stato effettivamente oltrepassato. Si crea così, all'interno della linea ufficiale di demarcazione, una zona di frontiera flessibile e indefinita.

La norma in questione vale lungo l'intero perimetro dei confini territoriali. Tuttavia, il respingimento differito è applicato, a Lampedusa e nelle sue acque, con particolare frequenza e nei confronti di numeri particolarmente elevati di persone. Dall'isola sono respinti con tale modalità sia i migranti intercettati subito dopo l'ingresso in acque territoriali (il confine marittimo è a 12 miglia dalla costa), sia i pochi che riescono a sbarcare autonomamente sull'isola, che sono così equiparati ai primi. Al riguardo è emblematico il commento rilasciato dal Sottosegretario dell'Interno Mantovano quando la direttiva europea sui rimpatri fa decadere alcune norme nazionali su detenzione e accompagnamento alla frontiera ${ }^{31}$. Mantovano, pur lamentando che tale circostanza renderà i rimpatri più complicati, precisa che l'allontanamento dei migranti non sarà invece pregiudicato a Lampedusa, poiché l'isola è una "zona di frontiera"32, dalla quale chi arriva irregolarmente non è rimpatriato ma, appunto, respinto. Proprio poche settimane prima il governo ha dato inizio ai respingimenti da Lampedusa verso la Tunisia - i primi dopo la Rivoluzione dei gelsomini ${ }^{33}$.

\section{Conclusioni}

Questo articolo ha mostrato come le politiche italiane di controllo dell'immigrazione abbiano determinato la "frontierizzazione" di Lampedusa e l'innalzamento del suo tasso di "confinità". II processo inizia con il ricorso

31 MASERA, Luca. II 'nuovo' art. 14 co. 5 ter d.lgs. 286/98 e la sua applicabilità nei procedimenti per fatti antecedenti all'entrata in vigore del d.I. 89/2011.

32 ILSOLE24ORE.COM. Mantovano: ma l'impianto non è saltato.

${ }^{33}$ Possono essere in parte equiparati alla categoria dei respingimenti differiti anche i respingimenti in Libia effettuati tra ottobre 2004 e gennaio 2006, e quelli effettuati dalle acque internazionali (ma transitando da Lampedusa) tra 2009 e 2010. 
generalizzato all'obbligo del visto e con l'introduzione delle sanzioni per i vettori. La scelta di costruire un centro di accoglienza/detenzione a Lampedusa, e le modalità della sua gestione, trasformano poi l'isola nel luogo elettivo di concentramento e trattenimento. La conseguente crescita dell'indotto, insieme all'inevitabile moltiplicazione degli operatori dell'immigrazione, sancisce definitivamente la metamorfosi di Lampedusa da semplice isola a quintessenza del confine, come conferma anche l'interpretazione che viene data del suo status giuridico territoriale. In due occasioni, poi, emergenze prodotte artificialmente provocano un repentino innalzamento della "confinità" di Lampedusa, fermando i trasferimenti dei migranti e determinando il sovraffollamento dell'isola. Infine, la "frontierizzazione" di Lampedusa consente di utilizzare l'isola come simbolo della frontiera anche "al contrario": lasciandola vuota, tra il 2009 e l'inizio del 2011.

Gli sviluppi più recenti appaiono però di segno opposto. Innanzitutto, il centro resta chiuso quasi un anno tra l'autunno 2011 e l'estate 2012. Questo spiega la riduzione degli arrivi nel 2012. Dopo la riapertura del centro Lampedusa riprende in pieno la propria funzione di frontiera, ma nel 2013 le cose cominciano a cambiare. Infatti, la percentuale degli arrivi in altre località siciliane e in altre regioni italiane aumenta, anche in relazione con l'aumento degli arrivi di profughi siriani e con le sempre più frequenti partenze di imbarcazioni dall'Egitto e dalla Turchia. A dicembre 2013, poi, il centro di detenzione dell'isola viene chiuso per ristrutturazione, per essere riaperto solo a ottobre 2014. Forse le autorità giudicano che continuare a innalzare la "frontierità" di Lampedusa non sia più conveniente. Fatto sta che per tutto il 2014 Lampedusa rimane pressoché esclusa dagli sbarchi, anche dopo la riapertura del centro. Le cose cominciano a cambiare nei primi mesi del 2015, benché Lampedusa resti comunque solo uno tra i tanti luoghi di primo approdo. II processo di costruzione materiale e simbolica della frontiera mediterranea è in trasformazione, e - mentre l'isola continua a fungere da sinonimo del "confine" - solo il tempo dirà se il "sistema" Lampedusa è ormai tramontato.

\section{Riferimenti bibliografici}

AIRL.IT. Comunicato congiunto. 1998. Disponibile su: <http://www.airl.it/ accorditrattati2.php>. Consultato il: 22.12.2014.

AIRL.IT. Processo verbale. 1998. Disponibile su: < http://www.airl.it/accorditrattati1. php>. Consultato il: 22.12.2014.

BOUBAKRI, Hassan. La Tunisia nel contesto euro-maghrebino. Controllo dei movimenti migratori e diritti dei migranti. In CUTTITTA, Paolo; VASSALLO PALEOLOGO, Fulvio (a cura di). Migrazioni, frontiere, diritti. Napoli: ESI, 2006, p. 41-64. 
CAMERA.IT. Comitato parlamentare Schengen-Europol. Sopralluogo a Lampedusa (18-19 e 20 luglio 1997). 1997. Disponibile su: <http://leg13.camera.it/ bicamerali/schengen/sopralluoghi/AGRIGENT.htm>. Consultato il: 22.12.2014.

CASSARINO, Jean-Pierre (a cura di). Unbalanced Reciprocities. Cooperation on Readmission in the Euro-Mediterranean Area. Washington: Middle East Institute, 2010. CASTRONOVO, Antonella. Dalla "Guerra dei disperati" alle navi galera. In MANNOIA, Michele (a cura di). Il silenzio degli altri: discriminati, esclusi, invisibili. Roma: XL edizioni, 2011, p. 83-94.

CUTTITTA, Paolo. Lo spettacolo del confine. Lampedusa tra produzione e messa in scena della frontiera. Milano-Udine: Mimesis, 2012.

CUTTITTA, Paolo. I confini d'Europa a Sud del Mediterraneo. Strumenti e incentivi per I'esternalizzazione dei controlli. In CUTTITTA, Paolo; VASSALLO PALEOLOGO, Fulvio (a cura di). Migrazioni, frontiere, diritti. Napoli: ESI, 2006, p. 13-40.

DEL GRANDE, Gabriele. Mamadou va a morire: La strage dei clandestini nel Mediterraneo. Due Santi di Marino: Infinito, 2007.

DELL'AGNESE, Elena. Geografia politica critica. Milano: Guerini, 2005.

EDELMAN, Murray. Constructing the Political Spectacle. Chicago: University of Chicago Press, 1992.

FORTRESSEUROPE.BLOGSPOT.IT. Deportati in Italia. La mano del ra'is dietro gli sbarchi. 2011. Disponibile su: <http://fortresseurope.blogspot.it/2011/05/ deportati-in-italia-dietro-gli-sbarchi.html>. Consultato il: 22.12.2014.

FRIESE, Heidrun. Border Economies. Lampedusa and the Nascent Migration Industry. Shima: The International Journal of Research into Island Cultures, v. 6, n. 2, 2012, p. 66-84.

ILSOLE24ORE.COM. Mantovano: ma l'impianto non è saltato. 2011. Disponibile su: <http://www.ilsole24ore.com/art/notizie/2011-04-29/mantovano-impiantosaltato-063804_PRN.shtml>. Consultato il: 22.12.2014.

LIBERTI, Stefano. A sud di Lampedusa: Cinque anni di viaggi sulle rotte dei migranti. Rome: Minimum Fax, 2008.

MASERA, Luca. II 'nuovo' art. 14 co. 5 ter d.Igs. 286/98 e la sua applicabilità nei procedimenti per fatti antecedenti all'entrata in vigore del d.I. 89/2011. 2011. Disponibile su: <http://www.penalecontemporaneo.it/upload/MASERA\%20 ART\%2014.pdf>. Consultato il: 22.12.2014.

MOUNTZ, Alison. Seeking Asylum: Human Smuggling and Bureaucracy at the Border. Minneapolis: University of Minnesota Press, 2010.

PAOLETTI, Emanuela. The Migration of Power and North-South Inequalities: The Case of Italy and Libya. Basingstoke: Palgrave Macmillan, 2010.

RODIER, Claire. Xénophobie business. A quoi servent les contrôles migratoires? Paris: La Découverte, 2012.

RONZITTI, Natalino. Il trattato Italia-Libia di amicizia, partenariato e cooperazione. Senato della Repubblica. Contributi di Istituti di ricerca specializzati, n. 108, gennaio/2009. 
SIMMEL, Georg. Sociologia. Torino: Comunità, 1998.

VAN HOUTUM, Henk; VAN NATERS, Ton. Bordering, Ordering and Othering. Tijdschrift voor Economische en Sociale Geografie, v. 93, n. 2, 2002, p. 125-136. VASSALLO PALEOLOGO, Fulvio. Diritti sotto sequestro. Roma: Aracne, 2012.

\section{Abstract}

\section{Lampedusa between border production and representation}

Why is Lampedusa a synonym for the Euro-African border? Borders are social products, and turning a place into a border, or transforming the symbolic and practical role of a border place, is always the result of specific social acts. Starting from this assumption, the paper analyses the most important acts that increased Lampedusa's "borderness", thus determining its "borderization", between the 1990s and 2011. The research focuses on specific choices of the Italian legislator, on the relations with North-African countries, and on the practices of border control and management: the establishment of a detention centre, the choice to systematically divert migrants to Lampedusa and to concentrate professionalized reception activities on the island, the production of artificial emergencies.

Keywords: Lampedusa, Italy, border, migration, control.

Articolo ricevuto il 04.02.2015

Accettato per la pubblicazione il 23.05.2015

Received for publication in February, 04 ${ }^{\text {th }}, 2015$.

Accepted for publication in May, 23 $3^{\text {th }}, 2015$.

ISSN impresso 1980-8585

ISSN eletrônico 2237-9843

http://dx.doi.org/10.1590/1980-85852503880004403 